\title{
Chronic low back pain patient groups in primary care - A cross sectional cluster analysis
}

\author{
Annika Viniol ${ }^{1 *}$, Nikita Jegan ${ }^{1}$, Oliver Hirsch ${ }^{1}$, Corinna Leonhardt ${ }^{1}$, Markus Brugger ${ }^{2,3}$, Konstantin Strauch $^{2,3}$, \\ Jürgen Barth ${ }^{4}$, Erika Baum ${ }^{1}$ and Annette Becker ${ }^{1}$
}

\begin{abstract}
Background: Due to the heterogeneous nature of chronic low back pain (CLBP), it is necessary to identify patient groups and evaluate treatments within these groups. We aimed to identify groups of patients with CLBP in the primary care setting.

Methods: We performed a k-means cluster analysis on a large data set $(n=634)$ of primary care patients with CLBP. Variables of sociodemographic data, pain characteristics, psychological status (i.e., depression, anxiety, somatization), and the patient resources of resilience and coping strategies were included.

Results: We found three clusters that can be characterized as "pensioners with age-associated pain caused by degenerative diseases", "middle-aged patients with high mental distress and poor coping resources", and "middle-aged patients who are less pain-affected and better positioned with regard to their mental health".

Conclusions: Our results supported current knowledge concerning groups of CLBP patients in primary care. In particular, we identified a group that was most disabled and distressed, and which was mainly characterized by psychological variables. As shown in our study, pain-related coping strategies and resilience were low in these patients and might be addressed in differentiating treatment strategies. Future studies should focus on the identification of this group in order to achieve effective treatment allocation.
\end{abstract}

Trial registration: German Clinical Trial Register DRKS00003123.

Keywords: Subgroups, Chronic low back pain, Primary care

\section{Background}

Chronic pain syndromes, especially chronic low back pain (CLBP) is one of the most prevalent diseases in Western populations [1] and is one of the most common reasons for primary care consultations [2]. CLBP is associated with a high burden of disease with respect to health care costs and patients' quality of life [3].

Current treatment recommendations emphasize a multidisciplinary approach for chronic lower back pain (CLBP) treatment [4]. This therapy approach is effective, but often unrealistic as it is organizationally challenging, expensive, and cannot be made accessible to all CLBP patients [5].

A possible solution could be considering whether every CLBP patient needs the multidisciplinary treatment.

\footnotetext{
* Correspondence: annika.viniol@staff.uni-marburg.de

'Department of General Practice / Family Medicine, Philipps University of Marburg, Karl-von-Frisch-Str. 4, 35043 Marburg, Germany

Full list of author information is available at the end of the article
}

Considering the heterogeneous and multifactorial nature of chronic pain patients, it might be necessary to differentiate the main characteristics of patient groups and evaluate individual treatment approaches [6].

Several attempts have been made to identify groups of pain patients. Studies are typically based on single dimensions such as traditional psychological measures (e.g., the Minnesota Multiphasic Personality Inventory or the Symptom Checklist-90-R) [7], pain expression [8], physical performance [9], patient perceived etiology [10], and psychophysiological responses [11]. Multifactorial analyses usually study the impact of psychosocial characteristics in conjunction with pain-related fear [12,13], emotional distress $[14,15]$, behavioral response [16,17], or other psychosocial and behavioral characteristics [18]. These studies are driven by prognostic risk factors; none focuses on protective factors like coping strategies

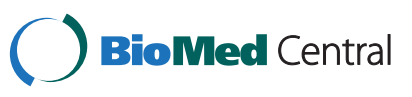


and self-efficacy, which influence chronic pain in a positive way.

We aimed to identify groups of patients with CLBP in the primary care setting. For group analyses, we considered pain characteristics, common psychological characteristics (i.e., depression, anxiety, somatization), and also included patient resources like resilience and coping strategies, which might contribute to future recommendations for CLBP patient counseling.

\section{Methods}

\section{Study design}

Our data referred to the cross sectional baseline analysis of a 12-month cohort study that identified risk factors and protective factors of pain generalization in primary care CLBP patients. A detailed study protocol has been published elsewhere [19].

This project is part of the research consortium LOGIN "Localized and Generalized Musculoskeletal Pain: Psychobiological Mechanisms and Implications for Treatment", funded by the German Federal Ministry of Education and Research.

\section{Study population}

During a 5-month period, fifty-eight general practitioners (evenly distributed in the northern region of Hessen in Germany) consecutively enrolled all eligible patients consulting for CLBP as a primary or secondary consulting reason (inclusion criteria). The symptom "chronic low back pain" was defined as back pain below the costal margin and above the inferior gluteal folds (with or without pain radiation), which had started at least three months prior and continued during most days (i.e., more than 50\%) in the last three months. Patients under 18 years, pregnant women, and persons with an insufficient understanding of the German language or severe cognitive impairments (e.g., dementia) were excluded from the study.

Patients who gave their informed consent were asked to complete a questionnaire directly after the consultation or at home. During the recruitment period, trained clinical monitors conducted two random quality control audits of the GPs' performance.

\section{Measurements}

To explore descriptive characteristics, the questionnaire included the following physical and psychological parameters (for detailed information please see Viniol et al. [19]).

\section{Pain characteristics and sociodemographic data}

To evaluate the number of different pain areas, we measured pain localization with the "body pain drawing model" proposed by Pfau et al. [20]. Pain anamnesis and sociodemographic data were determined with the
"German Pain Questionnaire", the official pain questionnaire of the German Association for the Study of Pain [21]. We used the following DGSS modules: duration, characteristics, course of pain, sociodemographic data, health care utilization, and medication.

We used the three-item social support subscale from the West Haven-Yale Multidimensional Pain Inventory (WHYMPI) to explore the partner's reaction in response to patient's pain (internal consistency of the subscales: $\alpha=0.63-0.90)[22,23]$.

The severity of chronic pain was measured by the German translation of von Korff's Graded Chronic Pain questionnaire (GCP) [24]. Severity is computed from "pain intensity" and "pain-related disability" (internal consistency of subscales: $\alpha=0.68-0.88$ ) [25].

\section{Comorbidities}

Using the Self-Administered Comorbidity Questionnaire (SACQ), we asked the patients about 14 common medical conditions: high blood pressure, heart disease, asthma, chronic obstructive pulmonary disease, ulcer/stomach disease, diabetes, high blood lipid level, kidney disease, osteoarthritis/degenerative arthritis, rheumatoid arthritis, osteoporosis, cancer disease, depression, other psychiatric diseases [26,27].

\section{Psychological parameters and patient resources}

Psychosomatic symptoms were measured with the somatization subscale of the Symptom Checklist 90-Revised (SCL-90-R), a commonly used psychological status symptom inventory for psychopathology (internal consistency: $\alpha=0.81$ ) [28].

Screening for anxiety disorders and depression was done by the Hospital Anxiety and Depression Scale (HADS) (internal consistency anxiety $\alpha=0.80$; depression $\alpha=0.81$ ) $[29,30]$.

Coping resources for back pain were evaluated by the FBR (Fragebogen zu Bewältigungsressourcen bei Rückenschmerzen) questionnaire from Tamcam et al. [31].

We used the resilience scale RS-11, a shortened and validated German form of the Wagnild \& Young questionnaire, to assess the resilience (internal consistency $\alpha=0.91)[32,33]$.

\section{Statistical analyses}

We performed k-means cluster analyses generalized to all scales of measurement with squared euclidean distances [34]. The k-means procedure identifies relatively homogenous groups while maximizing the variability between clusters. Variables with mixed scaling can be handled in cluster analysis [34,35]. Calculations were done with ALMO 15 (www.almo-statistik.de), which includes a k-means algorithm that is able to handle the different scalings of our variables and the large sample size. This 
program provides statistical measures to evaluate the appropriateness of a cluster solution (F-value, eta ${ }^{2}$ ) [35].

Cluster analysis is an iterative process looking for the most relevant variables adding to an interpretable solution [36,37]. Therefore, we ran several analyses for selection of variables, based on a variable-specific eta ${ }^{2}<$ 0.05 . Accordingly, the following variables were excluded: gender, living with a partner, applied for pension, pain distribution, medication, WHYMPI - social support scale, education level, kind of job, time of pain. We attached special importance to certain individual variables (number of pain areas, therapeutic strategies, consultations, operations) so that they were included irrespective of their eta ${ }^{2}$.

\section{Ethics statement}

The study was approved by the local ethics commission of Philipps University of Marburg, Germany (Ethik: 11.06.2010, AZ 88/10) and is in accordance with the Declaration of Helsinki.

\section{Results}

\section{Recruitment data}

Fifty-eight GPs with various practice characteristics (i.e., size, locations, and organizational structures) participated in the study. During the five-month recruitment period, they identified 746 eligible patients. Of these, 655 patients agreed to participate. A total of 647 subjects were analyzed; 8 patients were excluded because they did not report lower back pain in the pain drawing.

\section{Total group characteristics}

On average, the participating CLBP patients were 56 years old (SD 14.0; age range: $20-88$ years). The majority was female, married, and living in a two-person household. Just over half $(52.4 \%)$ reported having back pain for more than ten years. Half of the participants were employed; $74.6 \%$ of the unemployed participants were retired. Table 1 shows the characteristics of all recruited CLBP patients.

\section{Description of cluster modeling}

A three-cluster solution resulted in an $F_{\max }$-value of 76.16 and an eta ${ }^{2}$ of 0.191 , meaning that $19.1 \%$ of the variance can be explained by this partitioning and can be considered as a cluster solution with acceptable quality criteria [34,37,38].

The variables used for classification are depicted in Table 2. All included variables contributed substantially to the breakdown into the three clusters. Highest variablespecific eta ${ }^{2}$ values for the most important variables for cluster partitioning were found for employment status $\left(\right.$ eta $\left.^{2}=0.44\right)$, days of sick leave $\left(\mathrm{eta}^{2}=0.44\right)$, and age $\left(\right.$ eta $\left.^{2}=0.40\right)$. In addition, having a hobby/enjoyment as
Table 1 Sociodemographic data of the analyzed patients $(n=634)$

Variable

Sex [no. (\%)] $n=634$

Female

388 (61.2)

Age [mean: years (SD)] $n=634$

$56.30(13.95)$

Nationality [no. (\%)] $n=619$

German

$610(98.5)$

Other

$9(1.5)$

Marital status [no. (\%)] $n=634$

Single

$82(12.9)$

Married

$415(65.5)$

Divorced

$80(12.6)$

Widowed

$57(9.0)$

Living with a partner [no. (\%)] $n=611$

$477(78.1)$

Persons at household [no. (\%)] $n=605$

1 person

$87(14.4)$

2 persons

$297(49.1)$

3 persons

$114(18.8)$

4 persons

74 (12.2)

$>4$ persons

$33(5.4)$

Level and years of education [no. (\%)] $n=632$

$13 / 12$ years

$100(15.8)$

10 years

$191(30.2)$

9 years

$322(50.9)$

Other graduation

$15(2.4)$

No qualification

$4(0.6)$

Employment status [no. (\%)] $n=631$

Working (full or part-time)

$322(51.0)$

Reasons for not working [no. (\%)] $n=306$

Keeping house

$48(15.7)$

Retired

228 (74.6)

Unemployed

$27(8.8)$

Other

$3(1.0)$

First time of back pain [no (\%)] $n=634$

Since $<1$ year

77 (12.2)

Since $1-2$ years

$47(7.4)$

Since $2-5$ years

86 (13.6)

Since $5-10$ years

$92(14.5)$

Since $>10$ years

$332(52.4)$

a coping resource $\left(\right.$ eta $\left.^{2}=0.41\right)$, depression $\left(\right.$ eta $^{2}=0.31$ ), retirement status ( eta $\left.^{2}=0.35\right)$, and resilience $\left(\right.$ eta $^{2}=0.28$ ) were also found to play an important role in cluster partitioning. All variables used in the cluster analysis are listed in Table 2. 
Table 2 Contribution of classification variables to the separation of cluster $(n=634)$

\begin{tabular}{|c|c|c|}
\hline Variable & $\begin{array}{l}\mathrm{F}_{\max } \\
\text { value }\end{array}$ & $\begin{array}{c}\text { Variable } \\
\text { specific eta }\end{array}$ \\
\hline Age & 208.40 & 0.40 \\
\hline Number of persons at household & 39.18 & 0.12 \\
\hline \multicolumn{3}{|l|}{ Employment status } \\
\hline - Working (full or part-time) & 242.31 & 0.44 \\
\hline \multicolumn{3}{|l|}{ Reasons for not working } \\
\hline - Keeping house & 31.37 & 0.17 \\
\hline - Unemployed & 16.61 & 0.10 \\
\hline - Retired & 81.44 & 0.35 \\
\hline - Other & 5.38 & 0.03 \\
\hline Days on sick leave & 117.13 & 0.44 \\
\hline \multicolumn{3}{|l|}{ Degree of disability } \\
\hline - Yes & 56.83 & 0.16 \\
\hline Number of pain areas & 4.84 & 0.02 \\
\hline \multicolumn{3}{|l|}{$\begin{array}{l}\text { Index of the graded chronic } \\
\text { pain (von Korff) }\end{array}$} \\
\hline-0 & - & - \\
\hline-1 & 4.33 & 0.02 \\
\hline-2 & 18.85 & 0.06 \\
\hline-3 & 0.43 & 0.00 \\
\hline-4 & 25.73 & 0.08 \\
\hline \multicolumn{3}{|l|}{ Gut feeling: Will the pain get away? } \\
\hline - Yes & 33.29 & 0.10 \\
\hline $\begin{array}{l}\text { Number of different therapeutic } \\
\text { strategies }\end{array}$ & 19.81 & 0.06 \\
\hline $\begin{array}{l}\text { Number of consultations because of } \\
\text { back pain } / 6 \text { months }\end{array}$ & 12.14 & 0.04 \\
\hline $\begin{array}{l}\text { Number of operations because } \\
\text { of back pain }\end{array}$ & 14.33 & 0.05 \\
\hline Symptom check-list-90-R (Somatization) & 41.18 & 0.13 \\
\hline \multicolumn{3}{|l|}{$\begin{array}{l}\text { Self-administered comorbidity } \\
\text { questionnaire (SACQ) }\end{array}$} \\
\hline - Psychological comorbidities* & 23.87 & 0.09 \\
\hline - Musculoskeletal comorbidities ** & 56.47 & 0.19 \\
\hline - Other comorbidities *** & 30.61 & 0.11 \\
\hline Brief resilience scale (RS-11) & 118.04 & 0.28 \\
\hline \multicolumn{3}{|l|}{ Hospital anxiety and depression (HADS) } \\
\hline - Anxiety & 67.69 & 0.11 \\
\hline - Depression & 141.60 & 0.31 \\
\hline \multicolumn{3}{|l|}{ Coping resources of back pain (FBR) } \\
\hline - Emotional social support & 112.55 & 0.26 \\
\hline - Practical help & 85.62 & 0.22 \\
\hline - Exercise and relaxation & 101.90 & 0.25 \\
\hline - Hobby and enjoyment & 215.64 & 0.41 \\
\hline - Cognitive strategies & 183.63 & 0.37 \\
\hline
\end{tabular}

Table 2 Contribution of classification variables to the separation of cluster $(n=634)$ (Continued)

\begin{tabular}{lll}
\hline - Knowledge & 86.56 & 0.22 \\
- Spirituality & 63.15 & 0.17 \\
\hline
\end{tabular}

*Participants with the comorbidities depression and/or other psychiatric diseases.

**Participants with the comorbidities degenerative arthritis and/or rheumatoid arthritis and/or osteoporosis.

***Participants with the comorbidities high blood pressure and/or heart diseases and/or asthma and/or chronic obstructive pulmonary disease and/or ulcer/stomach diseases and/or diabetes and/or high blood lipid level and/or kidney diseases and/or cancer diseases.

\section{Description of clusters}

Table 3 depicts the description of the three clusters by means and proportions of classification variables.

\section{Cluster 1 - "pensioners with age-associated pain caused by} degenerative diseases"

( $\mathrm{n}=179,28.2 \%$ of all patients) comprised mainly retired persons with an average age of 68.2 years (SD 8.6; age range: $42-88$ years). Patients in this cluster suffered from a medium pain severity. In most cases, they had musculoskeletal and other chronic cardiovascular/pulmonary/ stomach diseases. In comparison to the other cluster groups, cluster 1 scored highest regarding perceived efficacy of coping resources and mental disorders. Furthermore, patients in cluster 1 were optimistic with regard to their pain prognoses.

\section{Cluster 2 - "middle-aged patients with high mental distress} and poor coping resources"

( $n=200,31.5 \%$ of all patients) consisted of middle-aged patients (mean 57.8 years, SD 13.1; age range: $26-87$ years) with only $44.2 \%$ employed. Most unemployed patients were pensioners due to disability (72.6\%). Remaining unemployed patients were either keeping house (17.7\%) or seeking work (8.8\%). Patients from cluster 2 had the most pronounced pain (highest rate of high pain severity and number of pain areas) and a high rate of pain-related disability. In comparison to the other clusters, patients in this cluster had more psychological distress (highest rate of the psychological comorbidities anxiety, depression, and symptoms of somatization). Furthermore, they had the worst resilience and the least efficient coping resources. They were high health care utilizers regarding the number of consultations and operations. We called them "middleaged patients with high mental distress and poor coping resources".

Cluster 3 - "middle-aged patients who are less pain-affected and better positioned with regard to their mental health" ( $\mathrm{n}=255,40.2 \%$ of all patients) was also comprised of middle-aged (mean 46.8 years; SD 10.3; age range: 20-73 


Clus

Cluster 1

(28.2\%)

Age [years: mean (SD)]

Persons at household [mean (SD)]

Employment status [no. (\%)]

- Working (full or part-time)

Reasons for not working [no. (\%)]

- Keeping house

- Unemployed\#

- Retired

- Other

Days on sick leave of the employed participants [mean (SD)]

Degree of disability [no. (\%)]

- Yes

Number of pain areas [mean (SD)]

Index of the graded chronic pain (von Korff) [no (\%)]

$-0$

$-1$

$-2$

$-3$

$-4$

Symptom check-list-90-R (Somatization) [mean (SD)]

Self-administered comorbidity questionnaire (SACQ) [no (\%)]

- Psychological comorbidities*

- Musculoskeletal comorbidities**

- Other comorbidities ${ }^{* *}$

Brief resilience scale (RS-11) [mean (SD)]

Hospital anxiety and depression (HADS) [mean (SD)]

- Anxiety

- Depression

$7.4(2.6)$

$13.9(5.0)$

$13.5(5.5)$

$14.2(4.4)$

$16.0(3.6)$

$15.7(3.9)$

$7.0(2.6)$

$6.2(3.3)$

$90(50.3)$

Cluster 2

$\mathrm{n}=200$

(31.5\%)

57.8 (13.1)

$2.2(0.1)$

88 (44.2)

$222(87.1)$

$11(6.7)$

$20(17.7)$

19 (57.9)

11 (33.3)

1 (3.0)

2 (6.1)

$4.8(8.2)$

20.9 (26.7)

$113(59.2)$

46 (18.6)

$4.4(2.2)$

$3.7(2.2)$

0 (0)

$0(0)$

$15(8.1)$

44 (18.0)

24 (13.0)

91 (37.1)

58 (31.4)

69 (28.2)

88 (47.6)

41 (16.7)

$14.4(7.2)$

$8.8(5.0)$

23 (19.5)

67 (44.4)

35 (15.2)

87 (57.6)

56 (24.3)

117 (77.5)

$125(54.3)$

47.3 (13.6)

$63.2(8.7)$

$7.0(3.3)$

$10.5(4.1)$

$6.8(2.4)$

$10.9(2.9)$

6.5 (4.6)

$11.6(5.2)$

7.5 (4.8)

$13.2(5.1)$

- Knowledge

- Spirituality

Gut feeling: Will the pain get away? [no (\%)]

- Yes
13.1 (4.7)

$14.7(4.0)$

$14.1(4.5)$

$8.2(4.2)$

$8.0(4.4)$

$7.8(4.3)$

$6.9(2.4)$

$3.8(3.5)$

$2.5(2.8)$

$73(36.5)$

40 (15.7) 
Table 3 Characterization of the three clusters of CLBP patients $(n=634)$ (Continued)

\begin{tabular}{|c|c|c|c|}
\hline Number of different therapeutic strategies [mean (SD)] & $7.1(3.8)$ & $6.6(3.9)$ & $5.0(3.4)$ \\
\hline Number of consultations because of back pain / 6 months [mean (SD)] & $5.7(5.9)$ & $6.1(7.2)$ & $3.6(4.2)$ \\
\hline Number of operations because of back pain [mean (SD)] & $0.3(0.8)$ & $0.5(1.4)$ & $0.1(0.3)$ \\
\hline
\end{tabular}

*Participants with the comorbidities depression and/or other psychiatric diseases.

**Participants with the comorbidities degenerative arthritis and/or rheumatoid arthritis and/or osteoporosis.

***Participants with the comorbidities high blood pressure and/or heart diseases and/or asthma and/or chronic obstructive pulmonary disease and/or ulcer/ stomach diseases and/or diabetes and/or high blood lipid level and/or kidney diseases and/or cancer diseases.

\#Proportion of unemployment among persons who are not working and are in an employable age ( $\leq 65$ years): Cluster 1: $15.4 \%$; Cluster 2: $20.0 \%$; Cluster 3: $36.7 \%$.

years), but mostly working $(87.1 \%)$ patients. In comparison to the second cluster, they were less pain affected, had fewer comorbidities and showed a better mental health (lower rates of anxiety, depression, and psychological comorbidities). Resilience was higher and coping resources were more pronounced than in patients belonging to cluster 2 . In addition, they rarely required health care.

Table 4 shows a summary of the main cluster characteristics.

\section{Discussion}

We performed a k-means cluster analysis on a large data set of primary care patients with chronic low back pain. We found three clusters that can be characterized as "pensioners with age-associated pain caused by degenerative diseases", "middle-aged patients with high mental distress and poor coping resources", and "middle-aged patients who are less pain-affected and better positioned with regard to their mental health".

Several researchers have stated the need to identify patient groups that could serve as target groups for effective treatment strategies [6]. Turk et al. identified three groups of chronic pain patients by the WHYMPI $[22,39]$. The first group, "dysfunctional patients", corresponds to patients with high pain severity, a low activity level, marked interference with everyday life due to pain, high affective distress, and low perception of life control. The second group, "adaptive copers", is characterized by a lower pain severity, a higher activity level, lower interference and affective distress, and higher life control. The third group, "interpersonally distressed", features middle pain severity, general activity, interference and affective distress, and lower social support than the other two groups.

Shaw et al. identified four groups of patients with acute work-related back pain based on disability risk factors (pain, depressive mood, fear avoidance beliefs, work inflexibility, and poor expectations for recovery) [40]. Group one consists of patients who are most affected by pain, concerned with high physical demands at work. This group resembles the "fear avoidance" category and shows low expectations of returning to work. Group two is characterized by a high rate of emotional distress and above average pain intensity. Patients in group three are identified by a high degree of concern about job placement. Finally, patients from group four show low risk factors for disability. They have positive expectations for workplace accommodation and returning to normal work.

Boersma et al. identified groups of acute and subacute spinal pain patients with regard to their risk for permanent pain or disability [41]. Their group profiles "fear-avoidant", "distressed fear-avoidant", "low risk" and "low risk depressed mood" are comparable to the results of Shaw et al.

Even though the included variables and patient populations (e.g., acute vs. chronic pain, low vs. no low back pain, different settings) of the aforementioned studies differ, they all have one aspect in common: All analyses revealed one patient group which seems most distressed and

Table 4 Main characteristics of the three clusters

\begin{tabular}{|c|c|c|c|}
\hline & Cluster 1 & Cluster 2 & Cluster 3 \\
\hline Age on average & 68 & 58 & 47 \\
\hline Working status of the majority & Pensioners & Invalidity pensioners & Working \\
\hline Severity of pain & Intermediate & High & Low \\
\hline Mental disorders (somatization, anxiety, depression, psychological comorbidities*) & Intermediate & High & Low \\
\hline Musculoskeletal ${ }^{* *} /$ other $^{* * * *}$ comorbidities & High & Low & Intermediate \\
\hline Resources (resilience, coping) & Intermediate & Low & High \\
\hline Health care utilization & Intermediate & High & Low \\
\hline
\end{tabular}

*Participants with the comorbidities depression and/or other psychiatric diseases.

**Participants with the comorbidities degenerative arthritis and/or rheumatoid arthritis and/or osteoporosis.

***Participants with the comorbidities high blood pressure and/or heart diseases and/or asthma and/or chronic obstructive pulmonary disease and/or ulcer/ stomach diseases and/or diabetes and/or high blood lipid level and/or kidney diseases and/or cancer diseases. 
shows above average mental distress and high pain severity. In this way, mental health status seems to be a key differential factor.

The primary care setting comprises a high prevalence of older, often multimorbid patients and many chronic diseases. Therefore, groups identified in different settings might not be relevant for general practice [42]. Even though we could confirm the presence of middle-aged groups with minor and major psychosocial distress, further studies are required; diagnostic studies to identify these groups and treatment studies, which would prove effectiveness of group-specific treatments.

Hill et al. developed the STarT Back tool for the primary care setting [43]. The tool classifies patients with low back pain (LBP) into three groups based on nine questions referring to potentially modifiable physical and psychological prognostic indicators for persistent, disabling symptoms. Patients are categorized as "low risk", "medium risk", and "high risk" for future disabling LBP. However, the studies from Hill et al. included patients with acute and chronic LBP. Our study focused only on patients with chronic LBP.

\section{Limitations}

Our study is subject to selection bias. GPs may have subconsciously preferred to recruit special cases (e.g., patients with higher disease severity or special personality), or forgotten to recruit patients due to high workload. Furthermore, some patients may have refused to participate in our study due to the long questionnaire, especially considering that our study population included a large proportion of older people with age-associated mental deficiencies. These factors might reduce the external validity of our results. In general, a limitation of cluster analysis is that the results depend on the input variables [44].

Since the less pain-affected patients of cluster three (average age: 46.8 years) are younger than the more pain-affected patients of cluster two (average age: 57.8 years), it is possible that younger patients from the third cluster will move to the second cluster as they age. This is especially likely because increased age is a proven risk factor for increased pain outcomes (e.g., transition from localized to widespread pain) [45]. We should soon be able to prove this hypothesis using follow-up data from our cohort study.

\section{Conclusion}

Our results supported current knowledge concerning groups of CLBP patients in primary care. This knowledge could be the starting point for developing a group-specific therapy for general practitioners.

For the group "pensioners with age-associated pain caused by degenerative diseases", a therapeutic orientation aligned with the guideline "pain of older people" [46,47] would be appropriate. It involves evidence-based treatment approaches individually adapted to older people and their comorbidities.

The group "patients of an employable age with high mental distress and poor coping resources" should receive multimodal pain therapy with a particular focus on psychotherapy to improve coping resources and resilience. With regard to the fact that mental disorders, especially depression, are the most decisive cost factor of chronic pain [48], it is particularly important to treat them in an effective manner.

For the group "patients of an employable age who are less pain-affected and better positioned with regards to their mental conditions", a general practitioner-based, time-contingent monitoring should be indicated. It should be planned independent of pain exacerbations and should be conducted according to the bio-psycho-social model of pain.

Next step of our research will be to develop a therapy study which evaluates the efficacy of group-specific therapy approaches.

\section{Abbreviations \\ CLBP: Chronic low back pain; GP: General practitioner; WHYMPI: West haven-uyale multidimensional pain inventory; GCP: Graded chronic pain; SACQ: Self-administered comorbidity questionnaire; SCL-90-R: Symptom checklist 90-revised; HADS: Hospital anxiety and depression scale; FBR: Fragebogen zu Bewältigungsressourcen bei Rückenschmerzen (questionnaire for assessing coping resources for back pain); RS: Resilience.}

\section{Competing interests}

Annika Viniol does not state any financial or non-financial conflicts of interest.

Nikita Jegan does not state any financial or non-financial conflicts of interest. Oliver Hirsch does not state any financial or non-financial conflicts of interest. Corinna Leonhardt does not state any financial or non-financial conflicts of interest.

Markus Brugger does not state any financial or non-financial conflicts of interest.

Konstantin Strauch does not state any financial or non-financial conflicts of interest.

Jürgen Barth does not state any financial or non-financial conflicts of interest. Erika Baum does not state any financial or non-financial conflicts of interest. Annette Becker was a consultant for Grünenthal $\mathrm{GmbH}$, from whom she has also received a speaker's fee.

\section{Authors' contributions}

AV planned the study, collected and analyzed data and wrote the manuscript. NJ collected and analyzed data. $\mathrm{OH}$ analyzed data. MB and KS gave statistical support and planned the study. JB and EB planned and revised study design. $C L$ and $A B$ planned the study, discussed the results and revised the manuscript critically. All authors edited the drafted version of the manuscript. All authors read and approved the final manuscript.

\section{Acknowledgements}

We thank our study nurses Muazzez Ilhan and Marion Herz-Schuchhardt for their excellent work. Furthermore, thank you to all participating doctors and patients. We also would like to thank Juliette Rautenberg for providing English-language editing, improving the precision and fluency of the manuscript.

\section{Funding}

This work is funded by the German Federal Ministry of Education and Research. 


\section{Author details}

'Department of General Practice / Family Medicine, Philipps University of Marburg, Karl-von-Frisch-Str. 4, 35043 Marburg, Germany. ${ }^{2}$ Institute of Medical Informatics, Biometry and Epidemiology, Chair of Genetic Epidemiology, Ludwig Maximilians University, Munich, Ingolstädter Landstraße 1, 85764 Neuherberg, Germany. ${ }^{3}$ Institute of Genetic Epidemiology, Helmholtz Center Munich - German Research Center for Environmental Health, Ingolstädter Landstraße 1, 85764 Neuherberg, Germany. ${ }^{4}$ Institute for Social and Preventive Medicine (ISPM), University of Bern, Niesenweg 6, 3012 Bern, Switzerland.

Received: 4 March 2013 Accepted: 24 September 2013

Published: 16 October 2013

\section{References}

1. Breivik H, Collett B, Ventafridda V, Cohen R, Gallacher D: Survey of chronic pain in Europe: prevalence, impact on daily life, and treatment. Eur J Pain 2006, 10:287-333.

2. Friessem $\mathrm{CH}$, Willweber-Strumpf $\mathrm{A}$, Zenz MW: Chronische Schmerzen in deutschen Facharztpraxen. Die aktuelle Inanspruchnahme des ambulanten Gesundheitssystems anhand der Prävalenz von Schmerzen. Schmerz 2010, 24:501-507.

3. Andersson GB: Epidemiological features of chronic low-back pain. Lancet 1999, 354:581-585.

4. Bundesärztekammer (BÄK), Kassenärztliche Bundesvereinigung (KBV), Arbeitsgemeinschaft der Wissenschaftlichen Medizinischen Fachgesellschaften (AWMF): Nationale Versorgungsleitlinie Kreuzschmerz - Langfassung. [National guideline for low back pain - long version]. Version 1.2. Berlin; 2011.

5. Dietl MKD: Versorgungssituation in der Schmerztherapie in Deutschland im internationalen Vergleich hinsichtlich Über-, Unter- oder Fehlversorgung: HTA-Bericht. Köln: [Health care services of pain therapy in Germany - an international comparison regarding to over-, undersupply and inappropriate health care services]; 2011.

6. Wand B, O'Connell N: Chronic non-specific low back pain - sub-groups or a single mechanism? BMC Musculoskelet Disord 2008, 9:11.

7. Schwimmer G, Robinson M, Geissner M: Relationship of MMPI cluster types, pain coping strategy, and treatment outcome. Clin J Pain 1992, 2:131-137.

8. Keefe FJ, Bradley LA, Crisson JE: Behavioral assessment of low back pain: identification of pain behavior subgroups. Pain 1990, 40:153-160.

9. Hutten MM, Hermens HJ, Zilvold G: Differences in treatment outcome between subgroups of patients with chronic low back pain using lumbar dynamometry and psychological aspects. Clin Rehabil 2001, 15:479-488.

10. Waylonis GW, Perkins RH: Post-traumatic fibromyalgia. A long-term follow-up. Am J Phys Med Rehabil 1994, 73:403-412.

11. Thieme K, Rose U, Pinkpank T, Spies C, Turk DC, Flor H: Psychophysiological responses in patients with fibromyalgia syndrome. J Psychosom Res 2006, 61:671-679.

12. Boersma K, Linton SJ: Expectancy, fear and pain in the prediction of chronic pain and disability: a prospective analysis. Eur J Pain 2006, 10:551-557.

13. Fritz JM, George SZ, Delitto A: The role of fear-avoidance beliefs in acute low back pain: relationships with current and future disability and work status. Pain 2001, 94:7-15.

14. Boersma K, Linton SJ: Psychological processes underlying the development of a chronic pain problem: a prospective study of the relationship between profiles of psychological variables in the fear-avoidance model and disability. Clin J Pain 2006, 22:160-166.

15. Pincus T, Burton AK, Vogel S, Field AP: A systematic review of psychological factors as predictors of chronicity/disability in prospective cohorts of low back pain. Spine (Phila Pa 1976) 2002, 27:109-120.

16. Thieme K, Flor H, Turk DC: Psychological pain treatment in fibromyalgia syndrome: efficacy of operant behavioural and cognitive behavioural treatments. Arthritis Res Ther 2006, 8:121.

17. Verra ML, Angst F, Brioschi R, Lehmann S, Keefe FJ, Staal JB, de Bie RA, Aeschlimann A: Does classification of persons with fibromyalgia into Multidimensional Pain Inventory subgroups detect differences in outcome after a standard chronic pain management program? Pain Res Manag 2009, 14:445-453.
18. Turk DC, Okifuji A, Sinclair JD, Starz TW: Pain, disability, and physical functioning in subgroups of patients with fibromyalgia. J Rheumatol 1996, 23:1255-1262.

19. Viniol A, Jegan N, Leonhardt C, Strauch K, Brugger M, Barth J, Baum E, Becker A: Study protocol: transition from localized low back pain to chronic widespread pain in general practice: identification of risk factors, preventive factors and key elements for treatment - A cohort study. BMC Musculoskelet Disord 2012, 13:77.

20. Pfau DB, Rolke R, Nickel R, Treede R, Daublaender M: Somatosensory profiles in subgroups of patients with myogenic temporomandibular disorders and fibromyalgia syndrome. Pain 2009, 147:72-83.

21. Deutsche Gesellschaft zum Studium des Schmerzes (DGSS): Deutscher Schmerzfragebogen. Version 2012.2. Colone; 2012

22. Kerns $R$, Turk D, Rudy $T$ : The West Haven-Yale multidimensional pain inventory (WHYMPI). Pain 1985, 23:345-356.

23. Flor H, Rudy TE, Birbaumer N, Streit B, Schugens MM: Zur Anwendbarkeit des West Haven-Yale Multidimensional Pain Inventory im deutschen Sprachraum. Daten zur Reliabilität und Validität des MPI-D. Schmerz 1990, 4:82-87.

24. von Korff M, Dworkin S, Resche L: Graded chronic pain status an epidemiologic evaluation. Pain 1990, 40:179-291.

25. Klasen BW, Hallner D, Schaub C, Willburger R, Hasenbring M: Validation and reliability of the German version of the chronic pain grade questionnaire in primary care back pain patients. Psychosoc Med 2004, 1:7.

26. Sangha O, Stucki G, Liang MH, Fossel AH, Katz JN: The self-administered comorbidity questionnaire. A new method to assess comorbidity for clinical and health services research. Arthritis Rheum 2003, 49:156-163.

27. Hüppe $\mathrm{A}$, Raspe $\mathrm{H}$ : Amplifizierte Rückenschmerzen und Komorbidität in der Bevölkerung. Der Schmerz 2009, 23:275-283.

28. Franke G, Stäcker K: Reliabilität und Validität der Symptom-Check-Liste (SCL-90-R; Derogatis 1986) bei Standardreihenfolge versus inhaltshomogener Itemblockbildung. Diagnostica 1995, 41:349-373.

29. Zigmont AS, Snaith RP: The hospital anxiety and depression scale. Acta Psychiatr Scand 1983, 67:361-370.

30. Herrmann C: International experiences with the hospital anxiety and depression scale - a review of validation data and clinical results. J Psychosom Res 1997, 42:17-41.

31. Tamcan Ö, Bantli S, Abel T, Barth J: Coping resources for back pain. a new assessment tool for clinical practice. Zeitschrift für Medizinische Psychologie 2010, 19:21-29.

32. Wagnild GM, Young HM: Development and psychometric evaluation of the resilience scale. J Nurs Meas 1993, 1:165-178.

33. Schumacher J, Leppert K, Gunzelmann T, Strauß B, Brähler E: Die Resilienzskala Ein Fragebogen zur Erfassung der psychischen Widerstandsfähigkeit als Personmerkmal. Z Klin Psychol Psychiatr Psychother 2005, 53:16-39.

34. Bacher J: Clusteranalyse. Anwendungsorientierte Einführung in Klassifikationsverfahren [Cluster analysis. Practical introduction in classification measures]. Oldenbourg: Munich; 2010.

35. Schendera CF: Clusteranalyse mit SPSS: Mit Faktorenanalyse. [Cluster analysis with SPSS: including factor analysis]. Oldenbourg: Munich; 2009.

36. Everitt BS: Cluster analysis. London: Arnold; 2001.

37. Kaufman L: Finding groups in data. An introduction to cluster analysis. Hoboken: Wiley-Blackwell Publishing Ltd; 2005.

38. Jaccard J: Statistics for the behavioral sciences. Belmont: Wadsworth; 2009

39. Turk DC, Rudy TE: Toward an empirically derived taxonomy of chronic pain patients: integration of psychological assessment data. J Consult Clin Psychol 1988, 56:233-238.

40. Shaw WS, Pransky G, Patterson W, Linton SJ, Winters T: Patient clusters in acute, work-related back pain based on patterns of disability risk factors. J Occup Environ Med 2007, 49:185-193.

41. Boersma K, Linton SJ: Screening to identify patients at risk: profiles of psychological risk factors for early intervention. Clin J Pain 2005, 21:38-43.

42. Hay EM, Dunn KM, Hill JC, Lewis M, Mason EE, Konstantinou K, Sowden G, Somerville S, Vohora K, Whitehurst D, Main CJ: A randomised clinical trial of subgrouping and targeted treatment for low back pain compared with best current care. The STarT Back Trial Study Protocol. BMC Musculoskelet Disord 2008, 9:58.

43. Hill JC, Dunn KM, Lewis M, Mullis R, Main CJ, Foster NE, Hay EM: A primary care back pain screening tool: identifying patient subgroups for initial treatment. Arthritis Rheum 2008, 59:632-641. 
44. Morrison DG: Measurement problems in cluster analysis. Management Science 1967, 13:775-780

45. Larsson B, Bjork J, Borsbo B, Gerdle B: A systematic review of risk factors associated with transitioning from regional musculoskeletal pain to chronic widespread pain. Eur J Pain 2012, 8:1084-1093.

46. AGS Panel on Persistent Pain in Older Persons: The management of persistent pain in older persons. J Am Geriatr Soc 2002, 50:205-224.

47. Hadjistavropoulos T: Self-management of pain in older persons: helping people help themselves. Pain Med 2012, 13:67-71.

48. Becker A: Health economics of interdisciplinary rehabilitation for chronic pain: does it support or invalidate the outcomes research of these programs? Curr Pain Headache Rep 2012, 16:127-132.

doi:10.1186/1471-2474-14-294

Cite this article as: Viniol et al:: Chronic low back pain patient groups in primary care - A cross sectional cluster analysis. BMC Musculoskeletal Disorders 2013 14:294.

\section{Submit your next manuscript to BioMed Central and take full advantage of:}

- Convenient online submission

- Thorough peer review

- No space constraints or color figure charges

- Immediate publication on acceptance

- Inclusion in PubMed, CAS, Scopus and Google Scholar

- Research which is freely available for redistribution 